\title{
CNS Region-Specific Oxytocin Receptor Expression: Importance in Regulation of Anxiety and Sex Behavior
}

\author{
Tracy L. Bale, ${ }^{1}$ Aline M. Davis, ${ }^{2}$ Anthony P. Auger, ${ }^{2}$ Daniel M. Dorsa, ${ }^{1}$ and Margaret M. McCarthy ${ }^{2}$ \\ ${ }^{1}$ Neurobiology and Behavior Program, Departments of Pharmacology and Psychiatry, University of Washington, Seattle, \\ Washington 98195, and 2Department of Physiology, University of Maryland, Baltimore, Maryland 21201
}

The oxytocin receptor (OTR) is differentially expressed in the CNS. Because there are multiple mechanisms by which the OTR can be transcriptionally induced, we hypothesized that differences in OTR expression may be explained by activation of distinct signal transduction pathways and may be critical for the control of anxiety and sex behaviors. To determine the regulation and functional significance of this expression, we infused female rats with modifiers of protein kinases before assaying for behavior and oxytocin receptor binding. In the ventromedial nucleus of the hypothalamus (VMH), estrogendependent induction of oxytocin receptors required protein kinase $\mathrm{C}$ activation, and oxytocin infused here promoted female sex behavior but had no effect on anxiety. In contrast, dopamine controlled tonic oxytocin receptor expression in the central nucleus of the amygdala (cAmyg) through activation of protein kinase $A$, and oxytocin infused here was anxiolytic but had no effect on female sex behavior. Therefore, we have identified brain region-specific regulation of the OTR in the $\mathrm{VMH}$ and cAmyg. Distinct signal transduction pathways regulating receptor expression and binding in each brain region may mediate in part the ability of oxytocin to exert these differential behavioral effects.

Key words: oxytocin; heterologous expression; amygdala; hypothalamus; lordosis; anxiety
The neuropeptide oxytocin (OT) is synthesized in neurons of the supraoptic and paraventricular (PVN) nuclei of the hypothalamus, which project to the posterior pituitary and release oxytocin into the bloodstream. This peripherally acting oxytocin is critical for the processes of lactation and parturition. Oxytocin neurons of the PVN also send projections to many regions within the brain, including the hippocampus, amygdala, and hypothalamus, in which oxytocin acts as a neurotransmitter involved in affiliative behaviors (Insel, 1992). Oxytocin receptors (OTRs) are found in many brain regions, including the central nucleus of the amygdala (cAmyg) and the ventromedial nucleus of the hypothalamus $(\mathrm{VMH})$ in which they are heterologously expressed (Bale et al., 1995a,b). These two brain regions are critical components of neural circuits regulating distinct behavioral responses. The VMH is an important mediator of female sex behavior (Pfaff et al., 1994), whereas the cAmyg is a portion of the limbic system that coordinates fear and anxiety responses (Davis, 1997; Roozendaal et al., 1997; LeDoux, 1998). The exhibition of rat female sex behavior requires estrogen action in the brain, including the induction of OTRs in the VMH (McCarthy et al., 1994; Bale and Dorsa, 1995a,b; Bale et al., 1995a,b). In contrast, OTR levels in the cAmyg are unaffected by estradiol (Bale and Dorsa, 1995a), despite dense collections of estrogen-concentrating neurons (Pfaff and Keiner, 1973).

In vitro, OTR expression is increased by activation of protein kinase A (PKA) or protein kinase C (PKC) (Bale and Dorsa,

\footnotetext{
Received Oct. 13, 2000; revised Jan. 2, 2001; accepted Jan. 23, 2001.

This work was supported by United States Public Health Service (USPHS) Grant NS-20311 to D.M.D., Molecular and Cellular Biology Training Grant T32-GM07270 to T.L.B., and USPHS Grant MH52716 to M.M.M. We thank Cong Xu for her excellent technical support.

Correspondence should be addressed to Dr. Tracy L. Bale, The Clayton Foundation for Peptide Biology, The Salk Institute, 10010 N. Torrey Pines Road, La Jolla, CA 92037. E-mail: bale@salk.edu.

Copyright (C) 2001 Society for Neuroscience $0270-6474 / 01 / 212546-07 \$ 15.00 / 0$
}

1998). Previously, we isolated and sequenced $4.5 \mathrm{~kb}$ of an upstream sequence of a rat genomic OTR clone from a rat testis library and identified crucial putative response elements, including a cAMP response element and several activator protein-1 (AP-1) sites (Bale and Dorsa, 1997). Because there are multiple mechanisms by which the OTR can be induced transcriptionally, we hypothesized that differences in OTR expression between the $\mathrm{VMH}$ and cAmyg may be explained by activation of distinct signal transduction pathways in these brain regions, and these may be critical for the control of anxiety and sex behaviors.

\section{MATERIALS AND METHODS}

Animals. Sprague Dawley female rats were obtained from Charles River (Kingston, NY) and housed under reversed light/dark cycle conditions (lights out at 10:00 A.M.) in the animal facility of the University of Maryland, Baltimore. All animals were ovariectomized under anesthesia [75 $\mathrm{mg}$ of ketamine $+2.5 \mathrm{mg}$ of acepromazine $/ \mathrm{kg}$ body weight (BW)] and allowed at least 1 week to recover before the experiment. All procedures were in accordance with the guidelines of the University of Maryland Institutional Animal Care and Use Committee.

Acute intracerebral infusions for oxytocin receptor binding. Animals were anesthetized ( $75 \mathrm{mg}$ of ketamine $+2.5 \mathrm{mg}$ of acepromazine $/ \mathrm{kg} \mathrm{BW}$ ) and placed into a stereotaxic apparatus for site-specific infusions using a $1 \mu \mathrm{l}$ Hamilton syringe with attached needle. Stereotaxic coordinates for infusion into the amygdala were $2.8 \mathrm{~mm}$ caudal to bregma, $3.75 \mathrm{~mm}$ lateral to the midline, and $8.00 \mathrm{~mm}$ below the surface of the brain. Coordinates for injections into the VMH were the same, except $0.75 \mathrm{~mm}$ lateral to the midline. These coordinates were based on Figure 29 of the Paxinos and Watson (1986) rat brain atlas. Bilateral inf usions $(1 \mu l$ per side) consisted of phorbol ester (200 nM; Sigma, St. Louis, MO), forskolin (10 $\mu \mathrm{M}$; Sigma), H89 (200 $\mu$; Calbiochem, La Jolla CA), bisindolylmalemide I (BIM, $200 \mu \mathrm{M}$; Calbiochem), or the dopamine D1 receptor antagonist $R(+)$-SCH-23390 hydrochloride (10 $\mu$ g; Research Biochemicals, Natick, MA). Infusions were $\sim 1 \mathrm{~min}$ in duration, and the injection cannula was left in place for an additional $30 \mathrm{sec}$ before removal. At completion of the infusion, the incision was closed with surgical staples; the animal was treated with antibiotics and an analgesic and injected subcutaneously with either $2 \mu \mathrm{g}$ of estradiol benzoate (EB) or oil vehicle in $0.1 \mathrm{cc}$ of sesame oil before being returned to its home cage. To evaluate the 
inhibitory effect of BIM on OTR expression in the VMH, EB treatment is necessary to induce a detectable level of OTRs in this brain region. No EB treatment is necessary for OTR expression in the cAmyg because endogenous levels are maximal without estrogen exposure, and therefore ovariectomized females were left untreated.

Oxytocin receptor binding. Eight hours after infusion, animals were anesthetized lightly with $\mathrm{CO}_{2}$ before decapitation. Brains were removed rapidly, frozen briefly in isopentane, and stored at $-80^{\circ} \mathrm{C}$ until sectioning. Brains were cut on a cryostat $(20 \mu \mathrm{m}$ thick), and sections were mounted onto subbed slides and frozen at $-80^{\circ} \mathrm{C}$ until binding was performed. Slides were thawed before being washed twice for $10 \mathrm{~min}$ in

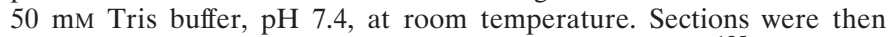
incubated in buffer containing: $50 \mathrm{~mm}$ Tris, $\mathrm{pH} 7.4,30 \mathrm{pM}{ }^{125}$ I-Ornithine Vasotocin (OTA; DuPont NEN, Boston, MA), $10 \mathrm{~mm} \mathrm{MgCl}_{2}, 0.1 \%$ BSA, and $0.05 \%$ bacitracin for $60 \mathrm{~min}$ at room temperature. Nonspecific binding was defined in adjacent sections that were exposed to both ${ }^{125} \mathrm{I}$-OTA and $1 \mu \mathrm{M}$ cold OT (Bachem, Torrance, CA). After the incubation period, slides were washed four times for $5 \mathrm{~min}$ each in a $50 \mathrm{~mm}$ Tris buffer containing $10 \mathrm{~mm} \mathrm{MgCl}_{2}$ at $4^{\circ} \mathrm{C}$. Slides were dipped rapidly in deionized water and dried under cool air. Sections were then exposed to film for $3 \mathrm{~d}$ before being developed by standard photographic methods. Autoradiography data analysis. Film autoradiographs were analyzed blind using a microcomputer-based image analysis system (MCID; Image Research, St. Catherines, Ontario, Canada). Oxytocin receptor binding optical density from autoradiographs was measured by subtracting nonspecific signal of adjacent sections from total signal to obtain the specific signal for each tissue section. The area of measurement for all samples was identical and was taken from the same region of the $\mathrm{VMH}$ or cAmyg as determined by choosing matching brain sections before analysis.

Repeated intracerebral infusions for behavioral testing. Animals were anesthetized ( $75 \mathrm{mg}$ of ketamine $+2.5 \mathrm{mg}$ of acepromazine $/ \mathrm{kg} \mathrm{BW}$ ), placed into a stereotaxic apparatus, and implanted with a bilateral guide cannula (22 gauge; Plastics One, Roanoke, VA) into either the VMH or cAmyg using the same stereotaxic coordinates as for the acute infusions. The guide cannula extended $7.5 \mathrm{~mm}$ into the brain, and the internal dummy cannula extended an additional $0.5 \mathrm{~mm}$. Animals were ovariectomized at the same time as the implantation of the cannula. At the time of infusion, awake animals were lightly restrained in a terry cloth towel, the dummy cannula was removed, and an injection cannula attached to a $1 \mu$ l Hamilton syringe via PE-10 tubing was inserted into the guide cannula. After infusions were performed in the same manner as described above, the fully awake animal was returned to its home cage.

Depending on the experiment, animals were infused with either the D1 antagonist SCH23390 $(10 \mu \mathrm{g})$, the PKC inhibitor BIM $(200 \mu \mathrm{M})$, or the PKC activator phorbol ester (PMA, $200 \mathrm{nM})$ in the morning ( 9:00 A.M.) and the evening ( 5:30 P.M.) on 2 consecutive days and again on the morning of the third day, for a total of five infusions. On the afternoon of the third day (the midpoint of the dark cycle), animals were infused with either oxytocin $(1 \mu \mathrm{g})$ or saline vehicle 10-20 min before behavioral testing.

Female sex behavior testing. Ovariectomized females were treated with EB (in sesame oil vehicle, $2-5 \mu \mathrm{g} / 0.1 \mathrm{cc}$, s.c.) 48 and $24 \mathrm{hr}$ before behavioral testing. At the midpoint of the dark cycle on the day of the test, animals were allowed to acclimate to a test arena before being tested manually for lordosis responsiveness by an investigator blinded to the particular treatment. Testing consisted of 10 trials of gently palpating the flanks and exerting pressure on the rump to induce a lordosis response. The degree of arching of the back and elevation of the rump was an indicator of the amplitude of the response and was assigned qualitative scores from zero (no lordosis) to three (maximal lordosis). Animals were pretested, infused with either oxytocin $(1 \mu \mathrm{g})$ or saline vehicle, and post-tested within 10-20 min of infusion. Because animal variability prevents absolute numbers for lordosis response, results were calculated as the difference for each animal between pretest and post-test scores. For this and all other behavioral tests, the experimenter scoring the response was blinded to the experimental group. All animals were treated and tested at the same time by the same investigators.

Open-field testing. At the midpoint of the dark phase of the cycle, animals were placed for $5 \mathrm{~min}$ in the center of an open-field apparatus made of clear Plexiglas (100 cm long, $80 \mathrm{~cm}$ wide, and $50 \mathrm{~cm}$ high); the floor of the apparatus was divided into 30 equally sized squares $\sim 16.5 \mathrm{~cm}$ per side. The movement of the animal was monitored such that each crossing over a line in the grid was noted. The squares around the perimeter were considered "outside," whereas all others were considered "inside." Data were expressed as the ratio of inside grid crossings to outside grid crossings. Because this was a test of novelty-induced anxiety, animals could not be pretested before drug treatment. Animals were tested in the apparatus $10-20 \mathrm{~min}$ after an infusion of oxytocin $(1 \mu \mathrm{g})$ or saline. An increase in the inside crossings/outside crossings ratio is an indicator of decreased anxiety. Animals tested for anxiety after infusion into the cAmyg were not pretreated with EB because OTRs in this brain region are maximal without estrogen exposure.

Elevated plus maze testing. Immediately after the open-field test, animals were placed in the center of a standard elevated plus maze (EPM) apparatus of $50 \mathrm{~cm}$ height with 50 -cm-long arms arranged perpendicularly. Two opposite arms were open, and two were enclosed with darkened Plexiglas walls. A $40 \mathrm{~W}$ bulb was suspended directly above the maze to provide even illumination to all arms. The number of entries and time spent in open and closed arms of the maze were recorded for $10 \mathrm{~min}$ with a digital recorder.

Histology. At the completion of the behavioral experiments, all animals were overdosed with Nembutal and perfused transcardially with ice-cold saline followed by $4 \%$ paraformaldehyde. Brains were removed, postfixed for $1 \mathrm{hr}$, cryoprotected in $30 \%$ sucrose, and stored frozen at $-80^{\circ} \mathrm{C}$ until sectioned on a cryostat. Sections $(50 \mu \mathrm{m}$ thick) were collected throughout the area of the cannula implant and stained with cresyl violet for verification of correct placement.

Statistics. Binding data and lordosis responding were assessed for statistical significance by ANOVA, and individual comparisons were made by post hoc Kruskal-Wallis test. Open-field data expressed as a ratio were assessed by the nonparametric Mann-Whitney $U$ test.

\section{RESULTS}

\section{Experiment I: Assessment of OTR binding by receptor autoradiography after manipulation of PKA or PKC by infusion into the VMH or cAmyg}

\section{Ventromedial hypothalamus}

Representative autoradiographs of oxytocin binding in the VMH after the various treatments are pictured in Figure 1. Quantification of binding showed that infusion of the PKC inhibitor, BIM $(200 \mu \mathrm{M})$, significantly decreased OTR binding levels in the VMH compared with saline vehicle-infused, ovariectomized estradiol-replaced females (ANOVA; $p<0.01 ; n=4$ ) (Fig. 2A). Infusion of a phorbol ester (PMA, $200 \mathrm{nM}$ ) to mimic activation of PKC induced a significant increase in OTR binding in the absence of estrogen, compared with saline vehicle-inf used ovariectomized controls $(p<0.001 ; n=4-5)$ (Fig. $2 A)$. Inf usion into the VMH of H89 (200 $\mu \mathrm{M} ; n=3)$ to inhibit PKA in ovariectomized estradiol-replaced females had no effect on OTR binding, nor did inf usion of forskolin (200 nM; $n=3)$ to enhance PKA in ovariectomized females (Fig. 2B)

\section{Central nucleus of the amygdala}

Representative autoradiographs of oxytocin binding in the cAmyg after the various treatments are pictured in Figure 3. Quantification of binding showed that infusion of H89, which inhibits PKA activity, significantly decreased OTR binding in this nucleus compared with vehicle-infused ovariectomized controls (ANOVA; $p<0.01 ; n=4$ ) (Fig. 4A). The cAmyg expresses dopamine D1 receptors that are positively coupled to adenylate cyclase and downstream activation of PKA. Infusion of the D1 antagonist SCH23390 significantly decreased OTR binding density in the cAmyg of ovariectomized females, compared with vehicle-inf used controls ( $p<0.01 ; n=3-4)$ (Fig. 4B). Infusion of forskolin, an activator of PKA, combined with SCH23390 reversed the effects of the D1 antagonist, resulting in a return to near basal levels of OTR binding that was significantly greater than the $\mathrm{SCH}$ antagonist alone $(p<0.05 ; n=3)$ (Fig. $4 B$ ). Infusion of the PKC inhibitor, BIM $(200 \mu \mathrm{M})$, was without effect on OTR binding in the cAmyg of ovariectomized females (vehicle, $0.295 \pm 0.07$; BIM, $0.261 \pm 0.09)$. 

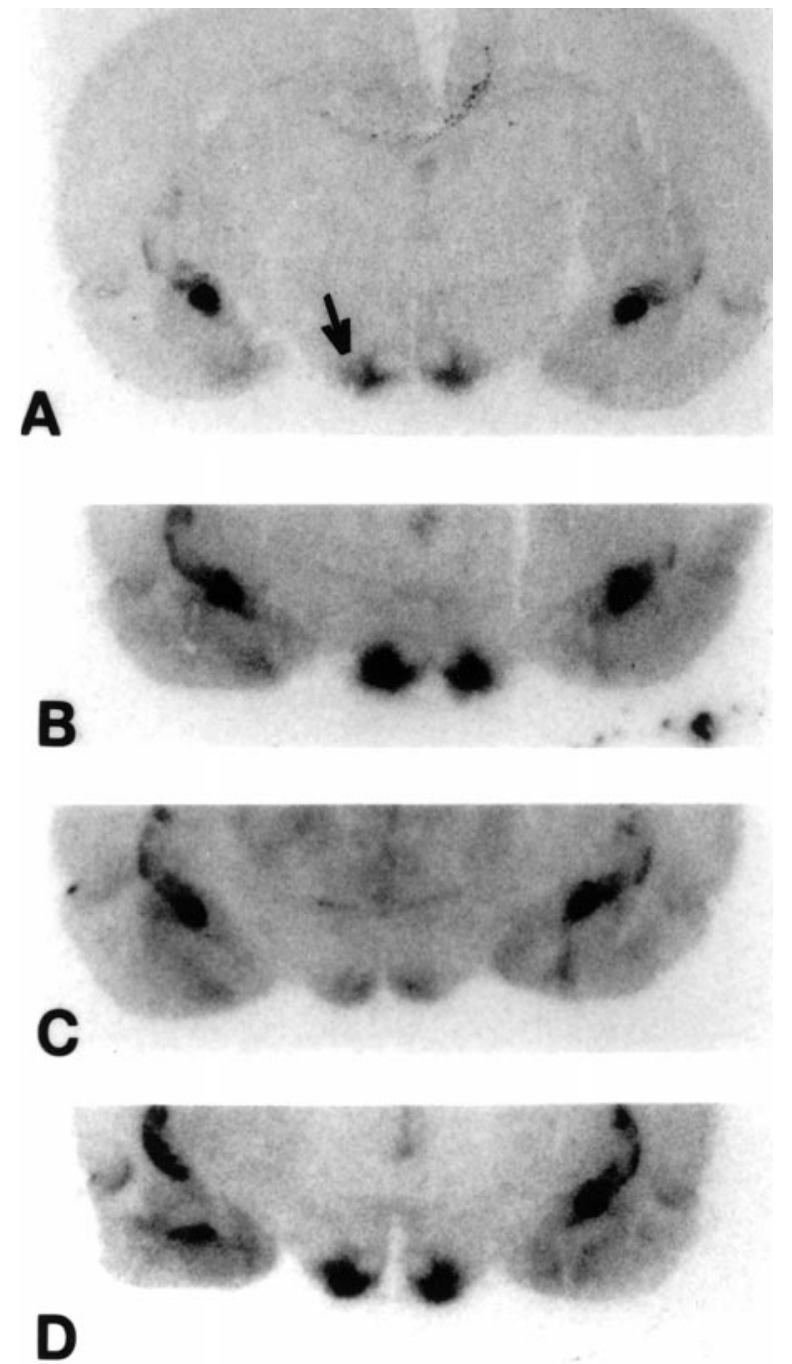

Figure 1. Representative autoradiographs illustrating oxytocin receptor binding in the VMH. Animals were pretreated with estradiol $(2 \mu \mathrm{g})$ or oil vehicle by subcutaneous injection; infused centrally, directly into the $\mathrm{VMH}$ with BIM (inhibitor of PKC) or the phorbol ester, PMA, $8 \mathrm{hr}$ before collection of the brains; and assayed for OTR binding by quantitative receptor autoradiography. Treatment groups were as follows: $A$, subcutaneous oil vehicle injection, saline vehicle infusion into the $\mathrm{VMH}$; $B$, subcutaneous estradiol injection, saline vehicle inf usion into the $\mathrm{VMH}$; $C$, subcutaneous estradiol injection, BIM infusion into the $\mathrm{VMH} ; D$, subcutaneous oil vehicle injection, PMA infusion into the VMH. Arrow indicates region of $\mathrm{VMH}$ that was analyzed.

\section{Experiment II: Assessment of site-specific behavioral effects of oxytocin infusion}

Female sex behavior

Oxytocin infused into the VMH of females primed with a low dose of estradiol significantly increased lordosis responding (oneway ANOVA with repeated measures post hoc Kruskal-Wallis; $p<0.001 ; n=9$ ) (Fig. $5 A$ ) but had no effect on lordosis when infused into the cAmyg $(p>0.5 ; n=8)$ (Fig. $5 B)$. The VMH oxytocin-induced increase in lordosis was blocked by previous treatment with the PKC inhibitor, BIM $(p<0.001)$ (Fig. $5 A)$. The ability of VMH-infused oxytocin to enhance lordosis responding was also evident in animals pretreated with the PKC activator PMA $(p<0.01)$, although there was no difference
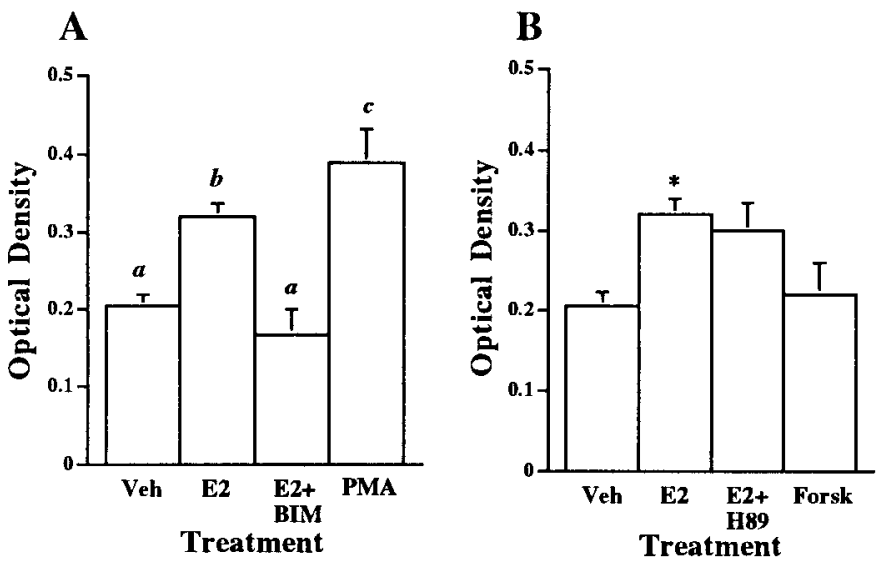

Figure 2. Quantification of OTR binding in the VMH. A, Optical density levels of OTR binding in the VMH of ovariectomized rats treated peripherally with estradiol $(E 2)$ or oil vehicle and centrally with inhibitors $(B I M)$ or activators $(P M A)$ of PKC. Treatment groups were Veh (subcutaneous oil vehicle injection, saline vehicle infusion into the $\mathrm{VMH} ; n=$ 4), $E 2$ [subcutaneous estradiol ( $2 \mu \mathrm{g})$ injection, saline vehicle inf usion into the VMH; $n=7$ ], E2 + BIM [subcutaneous estradiol injection, BIM (200 $\mu \mathrm{M})$ infusion into the VMH; $n=4$ ], or $P M A$ [subcutaneous oil vehicle injection, PMA (200 nM) infusion into the VMH; $n=5]$. Brains were collected $8 \mathrm{hr}$ after drug infusion and steroid treatment. Data were analyzed by one-way ANOVA and indicate a significant effect of treatment $\left[F_{(3,16)}=12.01 ; p<0.001\right]$; post hoc analysis indicates that $a$ is significantly different from $b(p<0.01)$ and $c(p<0.001)$. $B$, When compared with the same Veh- and E2-treated groups as above, infusion of forskolin (Forsk) (200 nM; $n=3)$, an activator of PKA, or H89 (E2 + H89) $(200 \mu \mathrm{M} ; n=3)$, an inhibitor of PKA, into the VMH of ovariectomized females was without effect on OTR binding in the VMH. All values are \pm SEM.

between animals pretreated with PMA or saline vehicle when they were given oxytocin (data not shown).

\section{Open-field test}

Oxytocin infusion into the cAmyg significantly increased openfield activity compared with controls, almost tripling the inside crossings/outside crossings compared with saline-infused animals (Mann-Whitney $U$ test; $p<0.01 ; n=9$ for oxytocin-inf used; $n=$ 6 for saline-infused) (Fig. $6 A$ ). The D1 antagonist SCH23390 blocked this effect of oxytocin ( $p<0.01 ; n=8)$ (Fig. 6A). In contrast, oxytocin infusion into $\mathrm{VMH}$ had no effect on open-field activity when compared with saline-infused controls (MannWhitney $U$ test; $p>0.8$ ) (Fig. 6B). There was no difference in the total number of crossings between any groups, indicating no changes in general locomotor activity $(\mathrm{VMH}$ oxytocin inf usion $=$ $126.2 \pm 49.4$; saline $=126.0 \pm 25.7$; cAmyg oxytocin infusion $=$ $112.3 \pm 11.6$; oxytocin $+\mathrm{SCH} 23390=123.4 \pm 14.1$; cAmyg saline $=137.7 \pm 19.8)$.

\section{Elevated plus maze}

Although infusion of oxytocin into the cAmyg appeared to increase the amount of time spent on the open arm of the plus maze and infusion of the D1 antagonist SCH23390 decreased the amount of time spent in the open arms when compared with oxytocin-infused controls $(\mathrm{SCH} 23390+$ oxytocin $=53.4 \pm 6.6$ sec, compared with oxytocin $=63.6 \pm 12.0 \mathrm{sec}$ and vehicle $=$ $50.3 \pm 11.3 \mathrm{sec}$ ), these differences failed to reach statistical significance. There were no differences in total arm entries when comparing oxytocin and saline infusion (oxytocin $+\mathrm{SCH} 23390=$ $14.4 \pm 2.8$; oxytocin $=10.9 \pm 1.8$; saline $=11.5 \pm 1.8)$, again indicating no changes in general locomotor activity as a result of 

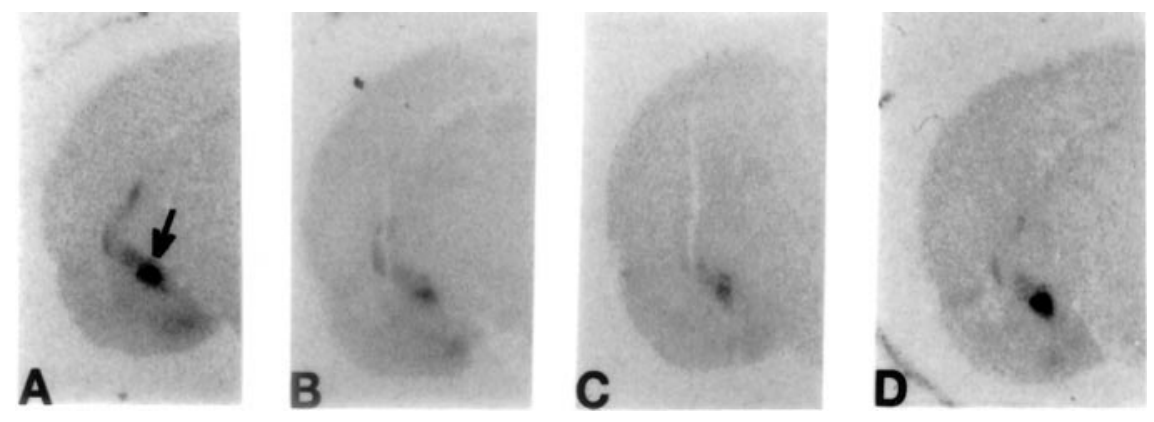

Figure 3. Representative autoradiographs illustrating OTR binding in the cAmyg. Animals were infused directly into the cAmyg, and brains were collected $8 \mathrm{hr}$ later and assayed for OTR binding by quantitative receptor autoradiography. Treatments were as follows: $A$, saline vehicle inf usion into the cAmyg; B, H89 (PKA inhibitor) infusion into the cAmyg; $C$, SCH23390 (dopamine D1 receptor antagonist) infusion into the cAmyg; or D, $\mathrm{SCH} 23390+$ forskolin infusion into the cAmyg. Arrow indicates region of cAmyg that was analyzed.

A

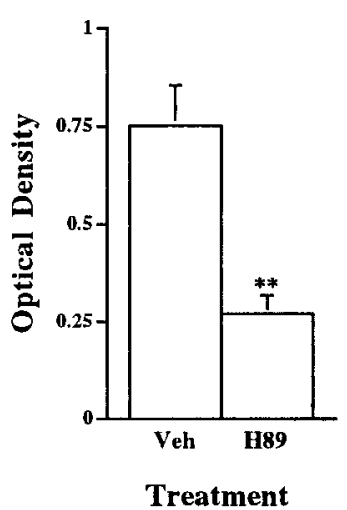

Figure 4. Quantification of OTR binding in the cAmyg. Ovariectomized rats were treated as follows: $A$, Veh 1 (saline vehicle for $\mathrm{H} 89$ inf usion into the cAmyg; $n=7$ ), and $H 89$ (H89 infusion into cAmyg; $n=5$ ); $B$, Veh 2 (saline vehicle for SCH23390 infusion into the cAmyg; $n=2$ ); $S C H$ (SCH23390 inf usion into the cAmyg; $n=3$ ), or $S C H+$ Forsk (SCH23390 + forskolin infusion into the cAmyg; $n=3$ ). Brains were collected $8 \mathrm{hr}$ after drug infusion. There was a significant effect of H89 infusion [ANOVA; $\left.F_{(1,10)}=13.96\right]$ and SCH23390 infusion $\left[F_{(3,7)}=10.62 ; p<\right.$ $0.01]$ when each was compared with its own controls. Post hoc analysis, $* * p<0.01$. All values are \pm SEM. Previous treatment with estradiol was found to have no influence on the effectiveness of H89 treatment (data not shown).

oxytocin infusion. Oxytocin inf usion into the VMH had no effect on EPM activity when compared with saline-infused controls (data not shown).

The location of the inf usion sites based on cresyl violet-stained sections resulted in the exclusion of one animal for cAmyg because of improper cannula placement.

\section{DISCUSSION}

We report here the regionally specific regulation of OTR binding and its significance in the control of anxiety and sex behaviors. In the female rat VMH, OTR binding is reduced by up to 200 -fold after ovariectomy and can be restored fully by replacement of estradiol (Johnson et al., 1989). A component of the estradiolinduced increase in OTRs involves increased levels of mRNA (Bale and Dorsa, 1995a,b), but whether this involves a genomic effect of estradiol is unknown. The mechanisms by which steroids can alter cellular functions have been expanded recently to include interactions with cytoplasmic signal transduction pathways (Zhou et al., 1996; Toran-Allerand et al., 1999). Our data indicate
A

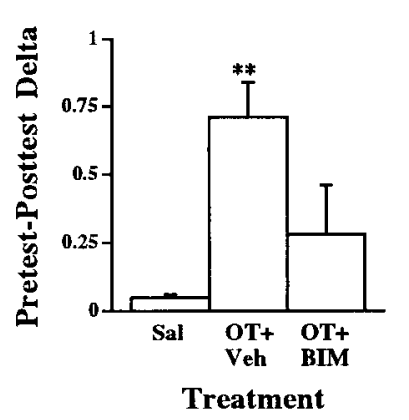

Figure 5. Effects of oxytocin infusion into the VMH and cAmyg on female sex behavior. Animals were primed with low doses of estradiol for $2 \mathrm{~d}$ and then pretested for lordosis responding before infusion of oxytocin $(O T ; 1 \mu \mathrm{g})$ or saline $(\mathrm{Sal})$ into the $\mathrm{VMH}$ or cAmyg. Post-testing was performed 10-20 min after infusion. Data are presented as the increase in lordosis amplitude between the pretest and post-test. $A$, For animals with indwelling cannulas in the $\mathrm{VMH}$, oxytocin infusion significantly increased lordosis responding compared with animals either infused with saline or pretreated for $2 \mathrm{~d}$ with the PKC inhibitor, BIM. ANOVA with post hoc Kruskal-Wallis; ${ }^{*} p<0.001 ; n=9$ for all groups. These results show that oxytocin infusion into the $\mathrm{VMH}$ increases lordosis behavior and this behavior is blocked by pretreatment with BIM. $B$, For animals with indwelling cannulas in the cAmyg, there was no difference in lordosis responding between those infused with oxytocin $(n=5)$ versus saline $\left[n=4 ; F_{(1,14)}=0.25 ; p>0.5\right]$. All values are \pm SEM.

that estradiol increases oxytocin receptor binding in the $\mathrm{VMH}$ via a PKC-dependent mechanism. Inhibition of PKC in the $\mathrm{VMH}$ blocked the ability of estradiol to increase OTR binding, whereas activation of PKC significantly increased OTR binding in the absence of estrogen. Therefore, the estradiol-induced increase in OTR binding in the VMH functions through activation of PKC and not through genomic actions of estrogen receptor directly at the OTR promoter.

In contrast to results in the VMH, OTR binding in the cAmyg is not regulated by estradiol (Bale et al., 1995a,b), and here we report that it is tonically maintained via a PKA-dependent mechanism involving the dopamine D1 receptor. This is the first evidence to suggest an interaction between the OTR and dopamine systems in the cAmyg. Inhibition of PKA in the cAmyg by H89 significantly decreased OTR binding when compared with controls. OTR binding was also reduced by antagonism of the dopamine D1 receptor by SCH23390. Tonic dopamine input into the cAmyg likely activates PKA through D1 receptors. The maintenance of OTR binding in the cAmyg appears specific to PKA 
A

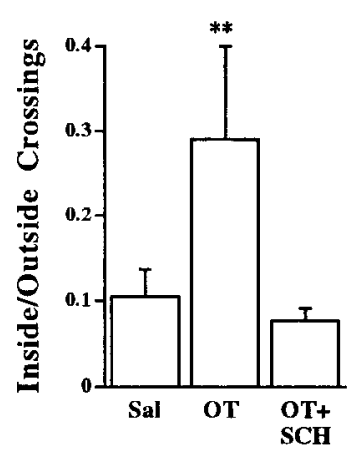

Treatment
B

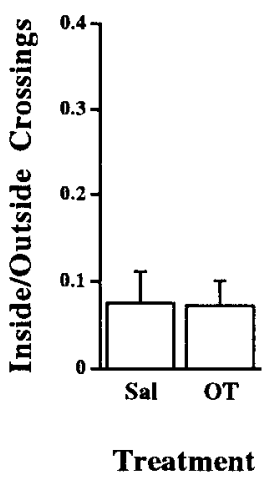

Figure 6. Effects of oxytocin infusion into the VMH and cAmyg on activity in an open field. Animals were placed into the center of an open-field testing apparatus within 10-20 min of infusion of oxytocin (1 $\mu \mathrm{g})$ or saline. The ratio of inside/outside crossings is a useful overall indicator of activity in an open field. An increase in the ratio indicates reduced anxiety-like behavior because it demonstrates that the animals are more willing to explore the center of the open field. $A$, For animals with indwelling cannulas in the cAmyg and infused with oxytocin $(n=9)$, there was a significant increase in the ratio when compared with salineinf used controls $(n=6)$. This effect was blocked by pretreatment with the dopamine D1 receptor antagonist, SCH23390 $(n=8)(* * p<0.01$; ANOVA). There were no differences in overall activity between any groups. $B$, For animals with indwelling cannulas in the $\mathrm{VMH}$, there was no effect of oxytocin infusion on activity in the open field.

because activation or inhibition of PKC had no effect at this brain site.

Oxytocin has been referred to as an "affiliative" hormone because of its intimate involvement in the regulation of behaviors involving attachment or close interaction between animals, including anxiety and sex behavior (Insel and Shapiro, 1992; McCarthy and Altemus, 1997). Consistent with previous reports (Schumacher et al., 1990; Schulze and Gorzalka, 1991), we found that infusion of oxytocin into the $\mathrm{VMH}$ increased female sex behavior as measured by lordosis responding. However, when PKC was inhibited, the enhancing effect of oxytocin on lordosis was blocked. The results of our binding studies suggest that the effect of PKC inhibition on lordosis may be caused by the consequent reduction of OTR expression in the $\mathrm{VMH}$, although we cannot rule out possible interactions with other components necessary for a lordotic response. We attempted to increase OTR levels by activation of PKC with a phorbol ester, with the prediction that oxytocin effects on lordosis would be enhanced. Although we continued to observe a significant increase in lordosis amplitude and frequency after oxytocin infusion, there was no statistical difference in oxytocin effectiveness between females pretreated with a phorbol ester versus saline. This is likely attributable to a maximal level of OTR expression after the estrogen treatment. Estrogen has pleiotropic effects on lordosis responding that are not limited to an effect via increased OTR binding (Pfaff et al., 1994). Nonetheless, our data are the first to demonstrate that oxytocin stimulation of lordosis behavior involves the activation of PKC to increase OTR transcription. Previous studies have shown that PKC activation in the VMH can increase female sex behavior (Kow and Pfaff, 1998) but have not implicated a specific neurotransmitter. Estradiol may directly activate the phosphatidylinositol second messenger pathway leading to PKC activation (Mobbs et al., 1989) or act indirectly by inducing the expression of PKC isoforms or fos and jun complexes that can then interact with $\mathrm{PKC}$ to stimulate transcription at AP-1 sites (Gaub et al., 1990) as are found in the OTR promoter (Bale and Dorsa, 1998). It cannot be overlooked that inhibition of PKC may also affect other processes within the $\mathrm{VMH}$ that may alter lordosis behavior.

In contrast to the $\mathrm{VMH}$, the cAmyg is a component of the limbic system and is involved in "emotional" responses, including fear and defensive behaviors (LeDoux, 1998). Oxytocin has been reported to have anxiolytic (McCarthy et al., 1996) and antidepressant (Arletti and Bertolini, 1987) actions, as well as facilitating pair-bonding (Williams et al., 1994; Insel and $\mathrm{Hu}-$ lihan, 1995) and mother-infant interactions (Pedersen and Prange, 1979; Fahrbach et al., 1984). Any or all of these may involve oxytocin action on the cAmyg. These behavioral responses are modulated by estradiol, but are not dependent on estradiol, as is the case for $\mathrm{VMH}$ regulation of female sex behavior. The exact CNS site(s) at which oxytocin acts to exert an anxiolytic effect is unknown. The cAmyg is one of the nuclei of the amygdala that receives convergent information from several other amygdaloid regions. It has been proposed that the afferents of this nucleus generate behavioral responses that reflect the sum of neuronal activity produced by the amygdala complex (Pitkanen et al., 1997). These observations led us to speculate that adaptive selective pressure has been in favor of a constitutive expression of OTRs in the cAmyg to allow for rapid responses to stressful or emotional stimuli by reducing anxiety. The cAmyg receives direct dopaminergic innervation from the midbrain, and neurons of this nucleus innervate the dopamine neurons of the substantia nigra (Rouillard and Freeman, 1995). Conditioned stressors increase the activity of dopamine neurons projecting to several components of the amygdala, including the central nucleus (Coco et al., 1992). Our findings suggest that one function of increased dopamine release to the cAmyg is the maintenance and/or increase of OTR expression levels. Oxytocin is also released centrally in response to a wide variety of stressors (Samson et al., 1985; Nishioka et al., 1998). Therefore, the oxytocin and dopaminergic systems may act in concert to reduce anxiety in response to social and environmental stressors. We found that oxytocin infusion into the cAmyg increased open-field activity almost threefold and that this effect was not present in animals pretreated with a D1 receptor antagonist. On the basis of our binding results, it is likely that the level of OTRs had been sufficiently reduced by pretreatment with the dopamine antagonist such that the oxytocin infused into the cAmyg could no longer exert an anxiolytic effect. Anxiety testing using the same treatments did not reach significance in the elevated plus maze. We believe that the open-field test is of more ethological relevance here because female rodents must leave their burrow and enter into an open field to mate. Oxytocin is strongly implicated in the control of social behaviors, suggesting that the difference in magnitude of the anxiolytic effect between the open field and elevated plus maze may be a function of measuring different types of anxiety and their potential social context. In contrast to the cAmyg, oxytocin infusion into the $\mathrm{VMH}$ had no effect on anxiety responses. To our knowledge, this is the first report of a site-specific anxiolytic effect of oxytocin.

A remaining question is the mechanism by which heterogeneous regulation of OTR expression is achieved in the cAmyg and VMH. The potential for an undiscovered second OTR gene in the cAmyg cannot be ruled out, and there is considerable 


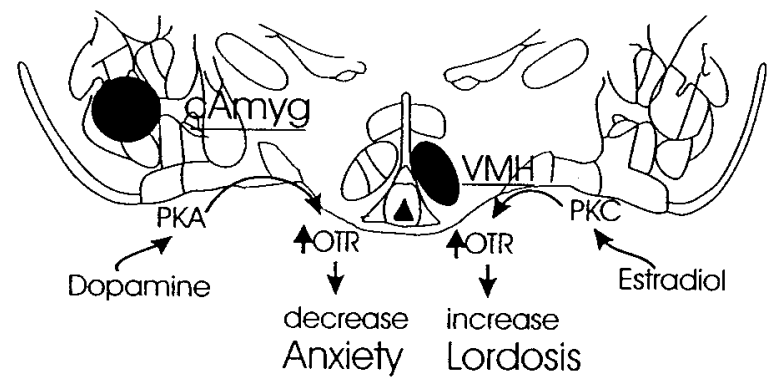

Figure 7. Summary diagram of effects observed on oxytocin receptor binding by estradiol and modulators of PKC and PKA and the resulting effects on behavior. Estradiol increases oxytocin receptor binding selectively in the VMN but not in the cAmyg. Estrogen action in the VMN involves PKC and results in increased female sex behavior as demonstrated by increased lordosis. Dopamine activation of the D1 receptor increases activation of PKA, resulting in increased or tonically maintained levels of oxytocin receptor binding in the cAmyg. Oxytocin acting in the cAmyg decreases anxiety. The use of distinct signal transduction pathways to regulate oxytocin receptor levels in the VMN and cAmyg may have evolved to allow for constitutive expression of oxytocin receptors in the cAmyg and an immediate response to possible life-threatening stimuli, as opposed to the hormonally constrained control of oxytocin receptors in the $\mathrm{VMN}$ that regulate female reproductive behavior.

circumstantial evidence to support this view (Bale et al., 1995a; Verbalis et al., 1995; Verbalis, 1999). An alternate hypothesis is based on the presence of three large TG-dinucleotide repeats in the OTR promoter (Bale and Dorsa, 1997). Submillimolar levels of calcium have been shown to regulate DNA structure at these TG-dinucleotide repeats in the other genes, causing an alteration in structure of the promoter and brain regionspecific expression of the protein (Dobi and v Agoston, 1998). The heterologous regulation of OTR expression in the cAmyg and VMH may be a function of differences in availability of response elements in the promoter caused by induction of secondary structure.

In summary, we have identified brain region-specific regulation of the OTR in the VMH and cAmyg and have demonstrated that this heterologous regulation is critical for two different behavioral effects of oxytocin. Although the estrogen-dependent OTR expression in the $\mathrm{VMH}$ is induced by activation of the PKC pathway, the receptors in the cAmyg are dependent on PKA and are tonically activated through dopamine D1 receptors in this nucleus. In the cAmyg, oxytocin acts to decrease anxiety, but in the $\mathrm{VMH}$ it acts to stimulate female sex behavior. A schematic representation of these results is depicted in Figure 7. Sex receptivity in the female rat is dependent on appropriate levels and duration of exposure to estradiol, thereby temporally restricting its expression to coincide with the event of ovulation. In contrast, behavioral responses to anxiety-provoking stimuli such as novelty require a continuously primed neural substrate to allow for rapid adaptation. OTRs in the $\mathrm{VMH}$ and cAmyg appear to regulate these different behaviors, respectively, and the distinct signal transduction pathways regulating receptor expression and binding in each brain region may mediate in part the ability of oxytocin to exert these differential effects.

\section{REFERENCES}

Arletti R, Bertolini A (1987) Oxytocin acts as an antidepressant in two animal models of depression. Life Sci 41:1725-1730.

Bale TL, Dorsa DM (1995a) Sex differences in and effects of estrogen on oxytocin receptor messenger ribonucleic acid expression in the ventromedial hypothalamus. Endocrinology 136:27-32.
Bale TL, Dorsa DM (1995b) Regulation of oxytocin receptor messenger ribonucleic acid in the ventromedial hypothalamus by testosterone and its metabolites. Endocrinology 136:5135-5138.

Bale TL, Dorsa DM (1997) Cloning, novel promoter sequence, and estrogen regulation of a rat oxytocin receptor gene. Endocrinology $138: 1151-1158$

Bale TL, Dorsa DM (1998) NGF, cyclic AMP, and phorbol esters regulate oxytocin receptor gene transcription in SK-N-SH and MCF7 cells. Mol Brain Res 53:130-137.

Bale TL, Dorsa DM, Johnston CA (1995a) Oxytocin receptor mRNA expression in the ventromedial hypothalamus during the estrous cycle. J Neurosci 15:5058-5064.

Bale TL, Pedersen CA, Dorsa DM (1995b) CNS oxytocin receptor mRNA expression and regulation by gonadal steroids. Adv Exp Med Biol 395:269-280.

Coco ML, Kuhn CM, Ely TD, Kilts CD (1992) Selective activation of mesoamygdaloid dopamine neurons by conditioned stress: attenuation by diazepam. Brain Res 590:39-47.

Davis M (1997) Neurobiology of fear responses: the role of the amygdala. J Neuropsychiatry Clin Neurosci 9:382-402.

Dobi A, v Agoston D (1998) Submillimolar levels of calcium regulates DNA structure at the dinucleotide repeat (TG/AC)n. Proc Natl Acad Sci USA 95:5981-5986.

Fahrbach SE, Morrell JI, Pfaff DW (1984) Oxytocin induction of shortlatency maternal behavior in nulliparous, estrogen-primed female rats. Horm Behav 18:267-286.

Gaub MP, Bellard M, Scheuer I, Chambon P, Sassone-Corsi P (1990) Activation of the ovalbumin gene by the estrogen receptor involves the fos-jun complex. Cell 63:1267-1276.

Insel TR (1992) Oxytocin-a neuropeptide for affiliation: evidence from behavioral, receptor autoradiographic, and comparative studies. Psychoneuroendocrinology 17:3-35.

Insel TR, Hulihan TJ (1995) A gender-specific mechanism for pair bonding: oxytocin and partner preference formation in monogamous voles. Behav Neurosci 109:782-789.

Insel TR, Shapiro LE (1992) Oxytocin receptor distribution reflects social organization in monogamous and polygamous voles. Proc Natl Acad Sci USA 89:5981-5985.

Johnson AE, Ball GF, Coirini H, Harbaugh CR, McEwen BS, Insel TR (1989) Time course of the estradiol-dependent induction of oxytocin receptor binding in the ventromedial hypothalamic nucleus of the rat. Endocrinology 125:1414-1419.

Kow LM, Pfaff DW (1998) Mapping of neural and signal transduction pathways for lordosis in the search for estrogen actions on the central nervous system. Behav Brain Res 92:169-180.

LeDoux J (1998) Fear and the brain: where have we been, and where are we going? Biol Psychiatry 44:1229-1238.

McCarthy MM, Altemus M (1997) Central nervous system actions of oxytocin and modulation of behavior in humans. Mol Med Today 3:269-275.

McCarthy MM, Kleopoulos SP, Mobbs CV, Pfaff DW (1994) Infusion of antisense oligodeoxynucleotides to the oxytocin receptor in the ventromedial hypothalamus reduces estrogen-induced sexual receptivity and oxytocin receptor binding in the female rat. Neuroendocrinology 59:432-440.

McCarthy MM, McDonald CH, Brooks PJ, Goldman D (1996) An anxiolytic action of oxytocin is enhanced by estrogen in the mouse. Physiol Behav 60:1209-1215

Mobbs CV, Rothfeld JM, Saluja R, Pfaff DW (1989) Phorbol esters and forskolin infused into midbrain central gray facilitate lordosis. Pharmacol Biochem Behav 34:665-667.

Nishioka T, Anselmo-Franci JA, Li P, Callahan MF, Morris M (1998) Stress increases oxytocin release within the hypothalamic paraventricular nucleus. Brain Res 781:56-60.

Paxinos G, Watson C (1986) The rat brain in stereotaxic coordinates. New York: Academic.

Pedersen CA, Prange Jr AJ (1979) Induction of maternal behavior in virgin rats after intracerebroventricular administration of oxytocin. Proc Natl Acad Sci USA 76:6661-6665.

Pfaff D, Keiner M (1973) Atlas of estradiol-concentrating cells in the central nervous system of the female rat. J Comp Neurol 151:121-158.

Pfaff DW, Schwartz-Giblin S, McCarthy MM, Kow L-M (1994) Cellular and molecular mechanisms of female reproductive behaviors. In: The physiology of reproduction (Knobil E, Neil E, eds), pp 107-220. New York: Raven.

Pitkanen A, Savander V, LeDoux JE (1997) Organization of intraamygdaloid circuitries in the rat: an emerging framework for understanding functions of the amygdala. Trends Neurosci 20:517-523.

Roozendaal B, Koolhaas JM, Bohus B (1997) The role of the central amygdala in stress and adaption. Acta Physiol Scand [Suppl] 640:51-54.

Rouillard C, Freeman AS (1995) Effects of electrical stimulation of the central nucleus of the amygdala on the in vivo electrophysiological activity of rat nigral dopaminergic neurons. Synapse 21:348-356. 
Samson WK, McDonald JK, Lumpkin MD (1985) Naloxone-induced dissociation of oxytocin and prolactin releases. Neuroendocrinology 40:68-71.

Schulze HG, Gorzalka BB (1991) Oxytocin effects on lordosis frequency and lordosis duration following inf usion into the medial pre-optic area and ventromedial hypothalamus of female rats. Neuropeptides 18:99-106.

Schumacher M, Coirini H, Pfaff DW, McEwen BS (1990) Behavioral effects of progesterone associated with rapid modulation of oxytocin receptors. Science 250:691-694.

Toran-Allerand CD, Singh M, Setalo Jr G (1999) Novel mechanisms of estrogen action in the brain: new players in an old story. Front Neuroendocrinol 20:97-121.
Verbalis JG (1999) The brain oxytocin receptor(s)? Front Neuroendocrinol 20:146-156.

Verbalis JG, Blackburn RE, Hoffman GE, Stricker EM (1995) Establishing behavioral and physiological functions of central oxytocin: insights from studies of oxytocin and ingestive behaviors. Adv Exp Med Biol 395:209-225

Williams JR, Insel TR, Harbaugh CR, Carter CS (1994) Oxytocin administered centrally facilitates formation of a partner preference in female prairie voles (Microtus ochrogaster). J Neuroendocrinol 6:247-250.

Zhou Y, Watters JJ, Dorsa DM (1996) Estrogen rapidly induces the phosphorylation of the cAMP response element binding protein in rat brain. Endocrinology 137:2163-2166. 